\title{
Cognitive Function and White Matter Lesions in Medication-Overuse Headache
}

\author{
Yue Xiang' \\ Shenggen Chen ${ }^{2}$ \\ Hanbin Lin $^{2}$ \\ Wenting Xiong ${ }^{2}$ \\ Zhenyang Zheng $\mathbb{D D}^{2}$ \\ 'Department of Nursing, Fujian Health \\ College, Fuzhou, 350I0I, People's \\ Republic of China; ${ }^{2}$ Department of \\ Neurology, Fujian Medical University \\ Union Hospital, Fuzhou, 35000I, People's \\ Republic of China
}

Purpose: This study was designed to investigate the cognitive function and the white matter lesions (WMLs) and the relationship between them in medication-overuse headache (MOH) patients.

Methods: Subjects were enrolled and performed Montreal Cognitive Assessment (MoCA, Chinese-Beijing Version), Hamilton Anxiety Rating Scale (HAMA), Hamilton Depression Rating Scale (HAMD-24), and Pittsburgh Sleep Quality Index (PSQI) to evaluate the general cognitive function, anxiety, depression and sleep quality, and they were divided into three groups according to the MoCA scores: healthy controls, $\mathrm{MOH}$ with normal cognition group and $\mathrm{MOH}$ with cognitive impairment group. All the participants underwent MRI scans and images were obtained for WML evaluation with Fazekas scale.

Results: One hundred thirty-four participants were enrolled into this study, 46 of them for healthy controls, and 88 for $\mathrm{MOH}$ patients, 40 of the $\mathrm{MOH}$ patients for $\mathrm{MOH}$ with cognitive impairment group, and 48 for $\mathrm{MOH}$ with normal cognition group. $\mathrm{MOH}$ patients had significantly lower MoCA scores, including the scores of visuospatial and executive function, attention, and orientation, while they had significantly greater HAMA scores, HAMD-24 scores, PSQI scores, and deep white matter hyperintensity scores compared to healthy controls. And in $\mathrm{MOH}$ patients, the age, disease duration, monthly headache days, and periventricular white matter hyperintensity scores in patients with cognitive impairment were greater than those in patients with normal cognition. Moreover, the MoCA scores were negatively related to age, disease duration, monthly headache days, and Fazekas scale scores, and disease duration and monthly headache days were significant predictors of cognitive impairment in $\mathrm{MOH}$ patients.

Conclusion: $\mathrm{MOH}$ patients showed cognitive impairment and increased WML burden. And in $\mathrm{MOH}$ patients, cognitive function was negatively related to WML burden, and disease duration and monthly headache days were potential predictors of cognitive impairment. Prompt and effective treatment to stop the progression of the disease may alleviate cognitive impairment in $\mathrm{MOH}$ patients.

Keywords: medication-overuse headache, cognitive function, white matter lesions, risk factor

\section{Introduction}

Medication-overuse headache $(\mathrm{MOH})$ is defined as a chronic headache that occurs on 15 or more days per month for more than 3 months in patients with a pre-existing primary headache caused by overuse of symptomatic headache medications, and the overall prevalence of $\mathrm{MOH}$ in the general population is about $1-2 \% .{ }^{1}$ According to the Global Burden of Disease Study 2015, MOH is the 20th cause of disability worldwide, which causes an impairment in the quality of life for sufferers and causes a heavy financial burden on society. $^{2}$
Correspondence: Zhenyang Zheng Department of Neurology, Fujian Medica University Union Hospital, Fuzhou, Fujian, People's Republic of China Tel +86-59l-83357896

Email zhenyangzheng@fjmu.edu.cn 
As a type of common chronic headache which developed from primary headache, $\mathrm{MOH}$ possessed longer disease duration and more attack frequencies. Neuroimaging studies indicated an increased prevalence of brain white matter lesions (WMLs) in migraine patients, ${ }^{3,4}$ and disease duration and headache attack frequency were indicators of WMLs in migraine. ${ }^{5,6}$ Moreover, our previous study suggested that WMLs were more prevalent in $\mathrm{MOH}$ patients. ${ }^{7}$

Cross-sectional studies showed that cognitive impairment was frequent in patients with chronic migraine and patients with chronic tension-type headache, ${ }^{8-10}$ and migraine and tension-type headache were also associated with non-vascular dementia. ${ }^{11}$ Our recent study found that the risk of cognitive decline was elevated in $\mathrm{MOH}$ patients. $^{12}$

WMLs were associated with impaired cognitive function, ${ }^{13}$ and the severity of WMLs at baseline was associated with the cognitive decline in the nondemented elderly over time. ${ }^{14}$ However, the relationship between cognitive function and WMLs in $\mathrm{MOH}$ patients was not yet clear. This study was performed to investigate the cognitive function and WMLs and the relationship between them in $\mathrm{MOH}$ patients.

\section{Methods}

\section{Participants}

This cross-sectional study was conducted between June 2016 and October 2019. The participants were recruited from the $\mathrm{MOH}$ patients attending the Department of Neurology, Fujian Medical University Union Hospital. Only patients who met all the following criteria were enrolled in the study: (a) diagnosis of $\mathrm{MOH}$ based on the third edition of the International Classification of Headache Disorders (beta version); ${ }^{15}$ (b) headache duration $\geq 1$ year; (c) without preventive treatment or detoxification before; (d) age between 18-80; (e) absence of dementia, including Alzheimer's disease, vascular dementia, and frontal temporal dementia, severe mental illness, neoplastic diseases, infectious diseases, rheumatic diseases or connective tissue diseases. Healthy subjects without a personal or family history of primary headache visiting our hospital for medical checkups during the study period were served as a healthy control group. All procedures performed in studies involving human participants were in accordance with the 1964 Helsinki declaration and its later amendments or comparable ethical standards. This study was approved by the Ethics
Committee of Fujian Medical University Union Hospital and informed consent was obtained from all individual participants included in the study.

\section{Baseline Information Collection}

The age, sex, years of schooling, accompanied hypertension, diabetes, smoking history, underlying primary headache, disease duration, and monthly headache days of the participants were investigated. Smoking history was defined as smoking at least 3 cigarettes per day on average for more than one year.

\section{Neuropsychology Assessment}

General cognitive function, anxiety, depression, and sleep quality were assessed with Montreal Cognitive Assessment (MoCA, Chinese-Beijing Version) (www.mocatest.org), Hamilton Anxiety Rating Scale (HAMA), ${ }^{16}$ Hamilton Depression Rating Scale (HAMD-24), ${ }^{17}$ and Pittsburgh Sleep Quality Index (PSQI), ${ }^{18}$ respectively by two trained physicians (Shenggen Chen and Hanbin Lin) blind to the clinical data. MoCA scores more than 25 (of 30), HAMA scores less than 7 (of 56), HAMD-24 scores less than 8 (of 76), and PSQI scores less than 7 (of 21) were classified as normal. According to the MoCA scores, the recruited $\mathrm{MOH}$ patients were divided into two groups, ie, patients with MoCA scores of more than 25 were classified into $\mathrm{MOH}$ with normal cognition group, and patients with MoCA scores of less than 26 were classified into $\mathrm{MOH}$ with cognitive impairment group.

\section{MRI Evaluation}

A 3.0-tesla MRI scanner (Prisma, Siemens Medical Systems, Erlangen, Germany) was employed to acquire images. The MRI protocol consisted of axial T1weighted images (TR/TE, $2500 \mathrm{~ms} / 10 \mathrm{~ms}$ ), axial T2weighted images (TR/TE, $4500 \mathrm{~ms} / 80 \mathrm{~ms}$ ), and coronal FLAIR images (TR/TE, $8000 \mathrm{~ms} / 100 \mathrm{~ms}$ ). For each image series, 35 slices covering the entire brain (matrix $224 \times$ 224; FOV $224 \mathrm{~mm} \times 224 \mathrm{~mm}$; slice thickness $4.0 \mathrm{~mm}$ without gap) were obtained.

WMLs were defined as hyperintense focal lesions on T2-weighted and FLAIR images and iso- or hypo-intense on T1-weighted images. WMLs were scored with Fazekas scale by two trained raters (Yue Xiang and Wenting Xiong) blind to the clinical data on a consensus basis. Fazekas scale scores were developed as a sum of periventricular white matter hyperintensity scores and deep white matter hyperintensity scores. Periventricular white matter 
hyperintensity was graded as $0=$ absence, $1=$ "caps" or pencil-thin lining, 2 = smooth "halo", 3 = irregular periventricular hyperintensity extending into the deep white matter. Deep white matter hyperintensity was graded as 0 = absence, 1 = punctate foci, 2 = beginning confluence of foci, 3 = large confluent areas. ${ }^{19}$

\section{Statistical Analysis}

Normality tests were performed to reveal the distribution of age, years of schooling, disease duration, monthly headache days, MoCA scores, HAMA scores, HAMD-24 scores, PSQI scores, and Fazekas scale scores before statistical analysis. For normally distributed data, they were expressed as the mean and standard deviation (SD), and the differences between groups were tested with independent samples $t$-test. For nonnormally distributed data, they were expressed as the median and inter-quartile range (IQR), and the differences between groups were tested with Mann-Whitney $U$-test. Differences in sex, accompanied hypertension, diabetes, and smoking history were tested with chi-square test. Correlations between MoCA scores and age, years of schooling, disease duration, monthly headache days, HAMA scores, HAMD-24 scores, PSQI scores, and Fazekas scale scores were analyzed using Spearman correlation analysis. Binary logistic regression models were used to estimate the risk factors for cognitive impairment in $\mathrm{MOH}$ patients. Odds ratios (ORs) and 95\% confidence intervals (CIs) were calculated. A two-tailed P-value $<0.05$ was considered statistically significant. All statistical analyses were performed using IBM SPSS Version 26.0 for Windows (SPSS Inc., Chicago, IL, USA).

\section{Results}

\section{Baseline Information}

Forty-six healthy controls (27 women, 19 men) and eightyeight $\mathrm{MOH}$ patients (49 women, 39 men) who met the inclusion criteria were enrolled into this study, 40 of the $\mathrm{MOH}$ patients for $\mathrm{MOH}$ with cognitive impairment group and 48 for $\mathrm{MOH}$ with normal cognition group. The drugs overused by $\mathrm{MOH}$ patients in our study included acetaminophen, ibuprofen, aminopyrine, phenacetin, diclofenac, loxoprofen, propyphenazone, aspirin, and combinations of simple analgesics. The age, sex, years of schooling, the incidence of hypertension, the incidence of diabetes, and the incidence of smoking history between healthy controls and $\mathrm{MOH}$ patients showed no significant difference (Table 1). The sex, years of schooling, the incidence of hypertension, the incidence of diabetes, the incidence of smoking history, and the incidence of migraine as primary headache between $\mathrm{MOH}$ patients with cognitive impairment and $\mathrm{MOH}$ patients with normal cognition showed no significant difference. However, the age, disease duration, and monthly headache days in $\mathrm{MOH}$ patients with cognitive impairment were greater than those in $\mathrm{MOH}$ patients with normal cognition (Table 2).

\section{Neuropsychology Assessment}

$\mathrm{MOH}$ patients had significantly lower MoCA scores compared to healthy controls, including the scores of visuospatial and executive function, attention, and orientation, while HAMA scores, HAMD-24 scores, and PSQI scores in $\mathrm{MOH}$ patients were greater than those in healthy controls (Table 3). The HAMA scores, HAMD-24 scores, and PSQI scores between $\mathrm{MOH}$ patients with cognitive impairment and $\mathrm{MOH}$ patients with normal cognition showed no significant difference (Table 4).

\section{WML Evaluation}

Fazekas scale showed that $\mathrm{MOH}$ patients had significantly greater deep white matter hyperintensity scores

Table I Demographic Characteristic of Study Participants

\begin{tabular}{|c|c|c|}
\hline \multirow[t]{2}{*}{ Characteristic } & Healthy Controls & MOH Patients \\
\hline & $(n=46)$ & $(n=88)$ \\
\hline Age, median (IQR), $y^{a}$ & $48.50(38.75-62.25)$ & $48.00(40.25-58.00)$ \\
\hline Women, No. (\%) & $27(58.70)$ & $49(55.68)$ \\
\hline Years of schooling, median (IQR), $y^{a}$ & $9.00(5.00-12.00)$ & $9.00(6.25-9.00)$ \\
\hline Hypertension, No. (\%) & $14(30.43)$ & $24(27.27)$ \\
\hline Diabetes, No. (\%) ${ }^{\mathrm{b}}$ & $10(21.74)$ & $21(23.86)$ \\
\hline Smoking history, No. (\%) ${ }^{\mathrm{b}}$ & $14(30.43)$ & $29(32.95)$ \\
\hline
\end{tabular}

Notes: ${ }^{\mathrm{a} M a n n}-\mathrm{Whitney} U$-test, $\mathrm{P}>0.05$. ${ }^{\mathrm{b}} \mathrm{C}$ i-square test, $\mathrm{P}>0.05$.

Abbreviations: $\mathrm{MOH}$, medication-overuse headache; IQR, inter-quartile range. 
Table 2 Demographic Characteristic of $\mathrm{MOH}$ Patients

\begin{tabular}{|c|c|c|}
\hline \multirow[t]{2}{*}{ Characteristic } & MOH Patients with Cognitive Impairment & MOH Patients with Normal Cognition \\
\hline & $(n=40)$ & $(n=48)$ \\
\hline Age, median (IQR), $y^{a}$ & $52.50(42.25-59.00)$ & $46.00(40.00-56.00)$ \\
\hline Women, No. (\%) & $22(55.00)$ & $27(56.25)$ \\
\hline Years of schooling, median (IQR), $y^{c}$ & $9.00(6.00-11.25)$ & $9.00(7.25-9.00)$ \\
\hline Hypertension, No. (\%) & $10(25.00)$ & $14(29.17)$ \\
\hline Diabetes, No. $(\%)^{\mathrm{b}}$ & $10(25.00)$ & II (22.92) \\
\hline Smoking history, No. (\%) & $13(32.50)$ & $16(33.33)$ \\
\hline Migraine as primary headache, No. $(\%)^{b}$ & $32(80.00)$ & $37(77.08)$ \\
\hline Disease duration, median (IQR), $y^{\mathrm{a}}$ & $10.00(8.00-14.75)$ & $6.00(5.00-8.00)$ \\
\hline Monthly headache days, median (IQR), $\mathrm{d}^{\mathrm{a}}$ & $25.00(23.00-27.00)$ & $18.50(\mid 7.00-21.75)$ \\
\hline
\end{tabular}

Notes: a Mann-Whitney U-test, $P<0.05$. ${ }^{b}$ Chi-square test, $P>0.05$. ${ }^{\mathrm{C}}$ Mann-Whitney U-test, $P>0.05$.

Abbreviations: $\mathrm{MOH}$, medication-overuse headache; $\mathrm{QRR}$, inter-quartile range.

Table 3 Neuropsychology Assessment in Study Participants

\begin{tabular}{|l|c|c|}
\hline Neuropsychology Assessment & Healthy Controls & MOH Patients \\
\cline { 2 - 3 } & $\mathbf{( n = 4 6 )}$ & (n = 88) \\
\hline MoCA scores, median (IQR) $^{\mathrm{a}}$ & $27.00(24.75-29.25)$ & $26.00(21.00-28.00)$ \\
Visuospatial and executive function, median (IQR) $^{\mathrm{a}}$ & $5.00(4.00-5.00)$ & $4.00(3.00-5.00)$ \\
Name, median (IQR) $^{\mathrm{b}}$ & $3.00(3.00-3.00)$ & $4.00(3.00-3.00)$ \\
Memory, median (IQR) $^{\mathrm{b}}$ & $4.00(3.00-5.00)$ & $5.00(3.00-5.00)$ \\
Attention, median (IQR) $^{\mathrm{a}}$ & $5.00(4.00-6.00)$ & $3.00(4.00-6.00)$ \\
Language, median (IQR) $^{\mathrm{b}}$ & $3.00(3.00-3.00)$ & $2.00(3.00-3.00)$ \\
Abstract ability, median (IQR) $^{\mathrm{b}}$ & $2.00(2.00-2.00)$ & $6.00(4.00-2.00)$ \\
Orientation, median (IQR) $^{\mathrm{a}}$ & $6.00(5.00-6.00)$ & $12.00(7.00-15.75)$ \\
HAMA scores, median (IQR) $^{\mathrm{a}}$ & $6.00(3.00-9.25)$ & $11.50(8.00-15.00)$ \\
HAMD-24 scores, median (IQR) $^{\mathrm{a}}$ & $4.00(2.00-9.00)$ & $12.00(5.00-15.00)$ \\
\hline PSQI scores, median (IQR) & $4.00(1.75-8.25)$ & \\
\hline
\end{tabular}

Notes: ${ }^{\mathrm{M}}$ Mann-Whitney U-test, $\mathrm{P}<0.05$. ' Mann-Whitney U-test, $\mathrm{P}>0.05$

Abbreviations: $\mathrm{MOH}$, medication-overuse headache; MoCA, Montreal Cognitive Assessment; IQR, inter-quartile range; HAMA, Hamilton Anxiety Rating Scale; HAMD, Hamilton Depression Rating Scale; PSQI, Pittsburgh Sleep Quality Index.

compared to healthy controls, while periventricular white matter hyperintensity scores between them showed no significant difference (Table 5). Periventricular white matter hyperintensity scores in
$\mathrm{MOH}$ patients with cognitive impairment were greater than that in $\mathrm{MOH}$ patients with normal cognition, while deep white matter hyperintensity scores between them showed no significant difference (Table 6).

Table 4 Neuropsychology Assessment in $\mathrm{MOH}$ Patients

\begin{tabular}{|l|c|c|}
\hline \multirow{2}{*}{ Neuropsychology Assessment } & MOH Patients with Cognitive Impairment & MOH Patients with Normal Cognition \\
\cline { 2 - 3 } & $\mathbf{( n = 4 0 )}$ & (n = 48) \\
\hline HAMA scores, median (IQR) & $12.00(7.25-16.50)$ & $11.00(6.25-15.75)$ \\
HAMD-24 scores, median (IQR) & $12.00(9.00-15.00)$ & $11.00(8.00-15.00)$ \\
PSQI scores, median (IQR) & $12.00(5.25-15.00)$ & $8.50(5.00-14.75)$ \\
\hline
\end{tabular}

Notes: ${ }^{a}$ Mann-Whitney U-test, $\mathrm{P}>0.05$.

Abbreviations: $\mathrm{MOH}$, medication-overuse headache; MoCA, Montreal Cognitive Assessment; IQR, inter-quartile range; HAMA, Hamilton Anxiety Rating Scale; HAMD, Hamilton Depression Rating Scale; PSQI, Pittsburgh Sleep Quality Index. 
Table 5 WMLs in Study Participants

\begin{tabular}{|l|c|c|}
\hline & Healthy Controls & MOH Patients \\
\cline { 2 - 3 } & $\mathbf{( n = 4 6 )}$ & $(\mathbf{n = 8 8 )}$ \\
\hline Fazekas scale scores, median (IQR) $^{\mathrm{a}}$ & $0(0-1.00)$ & $\mathrm{I}(0-2.00)$ \\
Periventricular white matter hyperintensity scores, median (IQR) $^{\mathrm{b}}$ & $0(0-1.00)$ & $\mathrm{I}(0-1.00)$ \\
Deep white matter hyperintensity scores, median (IQR) & $0(0-1.00)$ \\
\hline
\end{tabular}

Notes: a Mann-Whitney U-test, $P<0.05$. ' Mann-Whitney U-test, $P=0.05$.

Abbreviations: WMLs, white matter lesions; $\mathrm{MOH}$, medication-overuse headache; IQR, inter-quartile range.

Table 6 WMLs in MOH Patients

\begin{tabular}{|c|c|c|}
\hline & $\begin{array}{l}\text { MOH Patients with Cognitive } \\
\text { Impairment }\end{array}$ & $\begin{array}{c}\text { MOH Patients with Normal } \\
\text { Cognition }\end{array}$ \\
\hline & $(n=40)$ & $(n=48)$ \\
\hline Fazekas scale scores, median (IQR) ${ }^{\mathrm{a}}$ & $2.00(0.25-3.00)$ & $1.00(0-2.00)$ \\
\hline Periventricular white matter hyperintensity scores, median (IQR) & $1.00(0-2.00)$ & $0(0-1.00)$ \\
\hline Deep white matter hyperintensity scores, median (IQR) ${ }^{\mathrm{b}}$ & $1.00(0-1.00)$ & $0(0-1.00)$ \\
\hline
\end{tabular}

Notes: a Mann-Whitney U-test, $\mathrm{P}<0.05$. ' $\mathrm{Mann}-$ Whitney $U$-test, $\mathrm{P}>0.05$.

Abbreviations: WMLs, white matter lesions; $\mathrm{MOH}$, medication-overuse headache; IQR, inter-quartile range.

\section{Correlations Between MoCA Scores and Baseline Information, HAMA Scores, HAMD-24 Scores, PSQI Scores, and Fazekas Scale Scores in $\mathrm{MOH}$ Patients}

In $\mathrm{MOH}$ patients, MoCA scores were negatively related to age $(r=-0.315, P=0.003)$, disease duration $(r=-0.584, P<0.001)$, monthly headache days $(r=-0.494, P<0.001)$, and Fazekas scale scores $(r=-0.463, P<0.001$; Table 7).

\section{Risk Factors for Cognitive Impairment in $\mathrm{MOH}$ Patients}

Univariate logistic regression analysis revealed that age, disease duration, monthly headache days, and Fazekas scale scores were the independent risk factors for cognitive impairment in $\mathrm{MOH}$ patients $(P<0.05)$, while years of schooling, HAMA scores, HAMD-24 scores, PSQI scores, sex, accompanied hypertension, diabetes, smoking history, and underlying primary headache were not the independent risk factors $(P>0.05$; Table 8$)$. Therefore, four covariates, including age, disease duration, monthly headache days, and Fazekas scale scores, were included in our final multivariate logistic regression model to estimate the potential risk factors for cognitive impairment in $\mathrm{MOH}$ patients. Disease duration and monthly headache days were found to be significant predictors of cognitive impairment in $\mathrm{MOH}$ patients $(O R, 1.31 ; 95 \%$ $C I, 1.11-1.55 ; P=0.002$ and $O R, 1.42 ; 95 \% C I, 1.20$ $1.68 ; P<0.001$ respectively; Table 9).

\section{Discussion}

Our study indicated that $\mathrm{MOH}$ patients had significantly lower MoCA scores, especially in the domains of visuospatial and executive function, attention, and orientation, while they had significantly greater HAMA scores,

Table 7 Correlations Between MoCA Scores and Baseline Information, HAMA Scores, HAMD-24 Scores, PSQI Scores, and Fazekas Scale Scores in $\mathrm{MOH}$ Patients

\begin{tabular}{|l|c|c|c|c|c|c|c|c|c|}
\hline & & Age & $\begin{array}{c}\text { Years of } \\
\text { Schooling }\end{array}$ & $\begin{array}{c}\text { Disease } \\
\text { Duration }\end{array}$ & $\begin{array}{c}\text { Monthly } \\
\text { Headache Days }\end{array}$ & $\begin{array}{c}\text { HAMA } \\
\text { Scores }\end{array}$ & $\begin{array}{c}\text { HAMD-24 } \\
\text { Scores }\end{array}$ & $\begin{array}{c}\text { PSQI } \\
\text { Scores }\end{array}$ & $\begin{array}{c}\text { Fazekas Scale } \\
\text { Scores }\end{array}$ \\
\hline MoCA & $\mathrm{r}$ & -0.315 & 0.171 & -0.584 & -0.494 & 0.030 & 0.055 & -0.112 & -0.463 \\
scores & $\mathrm{P}$-value & 0.003 & 0.110 & $\mathrm{P}<0.001$ & $\mathrm{P}<0.001$ & 0.783 & 0.609 & 0.298 & $\mathrm{P}<0.001$ \\
\hline
\end{tabular}

Abbreviations: MOH, medication-overuse headache; MoCA, Montreal Cognitive Assessment; HAMA, Hamilton Anxiety Rating Scale; HAMD, Hamilton Depression Rating Scale; PSQI, Pittsburgh Sleep Quality Index. 
Table 8 Risk Factors for Cognitive Impairment in $\mathrm{MOH}$ Patients (Univariate Logistic Regression Analysis)

\begin{tabular}{|c|c|c|}
\hline Risk Factors & OR (95\% Cl) & P-value \\
\hline Age & $1.04(1.00-1.09)$ & 0.0497 \\
\hline Sex & $0.95(0.4 I-2.2 I)$ & 0.91 \\
\hline Years of schooling & $0.92(0.79-1.07)$ & 0.27 \\
\hline Hypertension & $0.8 \mathrm{I}(0.3 \mathrm{I}-2.09)$ & 0.66 \\
\hline Diabetes & $1.12(0.42-2.99)$ & 0.82 \\
\hline Smoking history & $0.96(0.39-2.35)$ & 0.93 \\
\hline Underlying primary headache & $1.19(0.43-3.32)$ & 0.74 \\
\hline Disease duration & $1.37(1.18-1.59)$ & $P<0.001$ \\
\hline Monthly headache days & $1.48(1.26-1.74)$ & $P<0.001$ \\
\hline HAMA scores & $1.02(0.96-1.09)$ & 0.55 \\
\hline HAMD-24 scores & $\mathrm{I} .02(0.94-\mathrm{I} . \mathrm{II})$ & 0.57 \\
\hline PSQI scores & $1.02(0.95-1.10)$ & 0.55 \\
\hline Fazekas scale scores & $1.94(1.29-2.90)$ & 0.001 \\
\hline
\end{tabular}

Abbreviations: MOH, medication-overuse headache; HAMA, Hamilton Anxiety Rating Scale; HAMD, Hamilton Depression Rating Scale; PSQI, Pittsburgh Sleep Quality Index; OR, odds ratio; $\mathrm{Cl}$, confidence interval.

Table 9 Risk Factors for Cognitive Impairment in $\mathrm{MOH}$ Patients (Multivariate Logistic Regression Model)

\begin{tabular}{|l|c|c|}
\hline Risk Factors & OR (95\% Cl) & P-value \\
\hline Disease duration & I.3I (I.II-I.55) & 0.002 \\
Monthly headache days & I.42 (I.20-I.68) & P < 0.00I \\
\hline
\end{tabular}

Abbreviations: $\mathrm{MOH}$, medication-overuse headache; OR, odds ratio; $\mathrm{Cl}$, confidence interval.

HAMD-24 scores, PSQI scores, and deep white matter hyperintensity scores compared to healthy controls. And in $\mathrm{MOH}$ patients, the age, disease duration, monthly headache days, and periventricular white matter hyperintensity scores in patients with cognitive impairment were greater than those in patients with normal cognition. Moreover, the MoCA scores were negatively related to age, disease duration, monthly headache days, and Fazekas scale scores, and disease duration and monthly headache days were significant predictors of cognitive impairment in MOH patients.

MOH patients demonstrated impaired general cognition as indicated by lowered MoCA scores, particularly in the domains of visuospatial and executive function, attention, and orientation. Altered volume in cerebellum and regions related to affection and cognitive processing (right lateral orbital gyrus), visual (left calcarine, bilateral middle occipital gyrus, right superior parietal lobe, optic chiasm), and auditory (right temporal transverse gyrus) perception was observed in $\mathrm{MOH}$ patients, ${ }^{20}$ which could be a morphological basis of cognitive impairment in $\mathrm{MOH}$.
Besides, MOH patients possessed prolonged P3 latency and lowered P3 amplitude, which could provide electrophysiological evidence for cognitive impairment in $\mathrm{MOH}^{21}$

$\mathrm{MOH}$ was considered to be associated with psychiatric comorbidities such as depression, anxiety, and insomnia, ${ }^{22}$ and these comorbidities could be risk factors in the evolution of migraine into $\mathrm{MOH}{ }^{23,24}$ Increasing headache frequency was associated with risk of occurrence of anxiety, depression, and insomnia, ${ }^{25}$ and higher migraine frequency was correlated with greater symptom scores of anxiety and depression. ${ }^{26} \mathrm{MOH}$ patients showed a high rate of depression and anxiety, which could negatively affect their headache attack, ${ }^{27}$ and depression and anxiety were found to be negative predictors in terms of treatment response in chronic migraine patients with or without medication-overuse. ${ }^{28-30}$ Moreover, lowered depression scores predicted a positive outcome of $\mathrm{MOH}$ detoxification. ${ }^{31}$ Furthermore, the volume of lower hippocampal subfields was negatively related to anxiety conditions in $\mathrm{MOH}$ patients. ${ }^{32}$

Recent studies suggested an increased prevalence of WMLs in migraine patients, especially deep WMLs, ${ }^{33}$ and our previous study showed that $\mathrm{MOH}$ patients had a greater prevalence of high WML load and they were at elevated risk of high deep WMLs load compared to healthy controls, ${ }^{7}$ which was in line with our finding that $\mathrm{MOH}$ patients possessed greater deep white matter hyperintensity scores compared to healthy controls.

We found that MoCA scores were negatively related to Fazekas scale scores in $\mathrm{MOH}$ patients, and periventricular white matter hyperintensity scores in patients with cognitive impairment were greater than that in patients with normal cognition. WMLs were implicated in the progression of cognitive impairment, and WMLs located at different regions, including periventricular and deep WMLs, evolved differently. A systematic review suggested that periventricular WMLs could have a significant negative impact on the cognition of older adults. ${ }^{34}$ To be more precise, frontal WMLs in the proximity of the frontal ventricles mainly affected executive function and parietotemporal WMLs in the proximity of the posterior horns deteriorated memory. ${ }^{35}$ Moreover, the frontal component of periventricular WMLs was associated with pronounced cortical atrophy, and the dorsal component of periventricular WMLs showed associations with the cognitive decline. $^{36}$ Additionally, periventricular WMLs were 
involved in the rate of cognitive decline. ${ }^{37}$ The underlying mechanisms of cognitive impairment caused by periventricular WMLs included impairment of nodal path length in the left opercular part of the inferior frontal gyrus, ${ }^{38}$ decreased regional cortical grey matter blood flow, ${ }^{39}$ disproportionate progressive hippocampal atrophy, ${ }^{40}$ and cortical atrophy. ${ }^{41}$

Age was associated with cognitive impairment among $\mathrm{MOH}$ patients. Age-related cognitive changes, including neuronal structure alterations, synapse loss, and neuronal network dysfunction result in brain structural and functional changes, and age-related diseases accelerate neuronal dysfunction, neuronal loss, and cognitive decline. ${ }^{42}$

Migraine has been linked to an increased prevalence of cognitive impairment, and the duration and frequency of migraine affect cognitive function. ${ }^{43}$ WMLs are more prevalent in migraine patients, and the disease duration and attack frequency have key roles in the formation of WMLs. ${ }^{6}$ WMLs may media the development of cognitive impairment in migraine. $\mathrm{MOH}$ patients had a high prevalence of cognitive impairment and WML burden, ${ }^{7}$ and the cognitive function was negatively related to WML burden. Our study indicated that the disease duration and headache frequency were the potential predictors of cognitive impairment in $\mathrm{MOH}$ patients. It could be assumed that longer disease duration and higher headache frequency in $\mathrm{MOH}$ could cause more serious WMLs, which would consequently lead to cognitive impairment. Further studies are needed to elucidate the mechanisms of the prevalence of cognitive impairment and WMLs in $\mathrm{MOH}$.

Several limitations in this study should be considered when interpreting the findings. Firstly, the cross-sectional survey of this study indicates associations between cognitive impairment and some risk factors. However, this study cannot determine whether these associations are causal. Additional longitudinal cohort studies will be needed to carry out the evaluations. Secondly, due to the strict inclusion criteria, a relatively small number of participants were enrolled into this study. Additional studies with a larger sample size are required to confirm our results.

\section{Conclusion}

$\mathrm{MOH}$ patients showed cognitive impairment and increased WML burden. And in $\mathrm{MOH}$ patients, cognitive function was negatively related to WML burden, and disease duration and monthly headache days were potential predictors of cognitive impairment. Our findings indicate that prompt and effective treatment to stop the progression of the disease may alleviate cognitive impairment in $\mathrm{MOH}$ patients.

\section{Acknowledgments}

This work was supported by grants from the Training Project for Young and middle-aged core talents in the Health system of Fujian province (grant No. 2017-ZQN -38), Natural Science Foundation of Fujian province (grant No. 2017J05126), and Sailing Fund Project of Fujian Medical University (grant No. 2016QH022).

\section{Disclosure}

The authors report no conflicts of interest in this work.

\section{References}

1. Headache Classification Committee of the International Headache Society (IHS). The international classification of headache disorders, 3rd edition. Cephalalgia. 2018;38(1):1-211. doi:10.1177/ 0333102417738202

2. Vos T, Allen C, Arora M, et al. Global, regional, and national incidence, prevalence, and years lived with disability for 310 diseases and injuries, 1990-2015: a systematic analysis for the Global Burden of Disease Study 2015. Lancet. 2016;388(10053):1545-1602.

3. Palm-Meinders IH, Koppen H, Terwindt GM, et al. Structural brain changes in migraine. JAMA. 2012;308(18):1889-1897. doi:10.1001/ jama.2012.14276

4. Kurth T, Mohamed S, Maillard P, et al. Headache, migraine, and structural brain lesions and function: population based epidemiology of vascular ageing-MRI study. BMJ. 2011;342:c7357. doi:10.1136/ bmj.c7357

5. Schmitz N, Admiraal-Behloul F, Arkink EB, et al. Attack frequency and disease duration as indicators for brain damage in migraine. Headache. 2008;48(7):1044-1055. doi:10.1111/j.15264610.2008.01133.x

6. Trauninger A, Leel-Ossy E, Kamson DO, et al. Risk factors of migraine-related brain white matter hyperintensities: an investigation of 186 patients. J Headache Pain. 2011;12(1):97-103. doi:10.1007/ s10194-011-0299-3

7. Zheng Z, Xiao Z, Shi X, et al. White matter lesions in chronic migraine with medication overuse headache: a cross-sectional MRI study. J Neurol. 2014;261(4):784-790. doi:10.1007/s00415-0147267-1

8. Qu P, Yu JX, Xia L, Chen GH. Cognitive performance and the alteration of neuroendocrine hormones in chronic tension-type headache. Pain Pract. 2018;18(1):8-17. doi:10.1111/papr.12574

9. Latysheva N, Filatova E, Osipova D, Danilov AB. Cognitive impairment in chronic migraine: a cross-sectional study in a clinic-based sample. Arq Neuropsiquiatr. 2020;78(3):133-138. doi:10.1590/0004$282 \times 20190159$

10. Ferreira KS, Teixeira CT, Cáfaro C, et al. Chronic migraine patients show cognitive impairment in an extended neuropsychological assessment. Arq Neuro Psiquiat. 2018;76(9):582-587. doi:10.1590/ 0004-282x20180085

11. Tzeng NS, Chung $\mathrm{CH}$, Lin FH, et al. Headaches and risk of dementia. Am J Med Sci. 2017;353(3):197-206. doi:10.1016/j. amjms.2016.12.014

12. Cai X, Xu X, Zhang A, et al. Cognitive decline in chronic migraine with nonsteroid anti-inflammation drug overuse: a cross-sectional study. Pain Res Manag. 2019;2019:7307198. doi:10.1155/2019/ 7307198 
13. Ashrafi F, Taheri MS, Farzaneh A, Behnam B, Ahmadi MA. Cognitive functions and white matter lesions on magnetic resonance images in a sample of normal Iranian population with cardiovascular risk factors. Neuroradiol J. 2019;32(2):108-114. doi:10.1177/ 1971400919825862

14. Qi X, Tang H, Luo Q, et al. White matter hyperintensities predict cognitive decline: a community-based study. Can J Neurol Sci. 2019;46(4):383-388. doi:10.1017/cjn.2019.47

15. Ettlin DA. The international classification of headache disorders, 3rd edition (beta version). Cephalalgia. 2013;33(9):629-808. doi:10.1177/0333102413485658

16. Hamilton M. Development of a rating scale for primary depressive illness. Br J Soc Clin Psychol. 1967;6(4):278-296. doi:10.1111/ j.2044-8260.1967.tb00530.x

17. Hamilton M. A rating scale for depression. J Neurol Neurosurg Psychiatry. 1960;23:56-62. doi:10.1136/jnnp.23.1.56

18. Buysse DJ, Reynolds CR, Monk TH, Berman SR, Kupfer DJ. The Pittsburgh sleep quality index: a new instrument for psychiatric practice and research. Psychiatry Res. 1989;28(2):193-213. doi:10.1016/0165-1781(89)90047-4

19. Fazekas F, Chawluk JB, Alavi A, Hurtig HI, Zimmerman RA. MR signal abnormalities at $1.5 \mathrm{~T}$ in Alzheimer's dementia and normal aging. AJR Am J Roentgenol. 1987;149(2):351-356. doi:10.2214/ ajr.149.2.351

20. Chen X, Chen Z, Dong Z, Liu M, Yu S. Morphometric changes over the whole brain in caffeine-containing combination-analgesic-overuse headache. Mol Pain. 2018;14:2070384879. doi:10.1177/ 1744806918778641

21. Xu C, Liu H, Wen Q, Wang R. Clinical research of the cognitive function and event-related potential P300 in patients with medication-overuse headache. Chin J Diagn. 2014;2(1):58-60.

22. Diener HC, Holle D, Solbach K, Gaul C. Medication-overuse headache: risk factors, pathophysiology and management. Nat Rev Neurol. 2016;12(10):575-583. doi:10.1038/nrneurol.2016.124

23. Radat F, Creac'H C, Swendsen JD, et al. Psychiatric comorbidity in the evolution from migraine to medication overuse headache. Cephalalgia. 2005;25(7):519-522. doi:10.1111/j.1468-2982.2005.00910.x

24. Viana M, Bottiroli S, Sances G, et al. Factors associated to chronic migraine with medication overuse: a cross-sectional study. Cephalalgia. 2018;38(14):2045-2057. doi:10.1177/0333102418761047

25. Buse DC, Reed ML, Fanning KM, et al. Comorbid and co-occurring conditions in migraine and associated risk of increasing headache pain intensity and headache frequency: results of the migraine in America symptoms and treatment (MAST) study. J Headache Pain. 2020;21(1):23. doi:10.1186/s10194-020-1084-y

26. Chu HT, Liang CS, Lee JT, et al. Associations between depression/ anxiety and headache frequency in migraineurs: a cross-sectional study. Headache. 2018;58(3):407-415. doi:10.1111/head.13215

27. Migliore S, Paolucci M, Quintiliani L, et al. Psychopathological comorbidities and clinical variables in patients with medication overuse headache. Front Hum Neurosci. 2020;14:571035. doi:10.3389/ fnhum.2020.571035

28. Schiano DCF, Caratozzolo S, Liberini P, Rao R, Padovani A. Response predictors in chronic migraine: medication overuse and depressive symptoms negatively impact onabotulinumtoxin-a treatment. Front Neurol. 2019;10:678. doi:10.3389/fneur.2019.00678
29. Disco C, Bellamio M, Fuccaro M, et al. O051. Chronic migraine and onabotulinumtoxin A: a prospective study on patients treated at the Headache Centre of the Padua University and analysis of possible predictors of responsivity. J Headache Pain. 2015;16(Suppl 1):A98. doi:10.1186/1129-2377-16-S1-A98

30. Probyn K, Bowers H, Caldwell F, et al. Prognostic factors for chronic headache: a systematic review. Neurology. 2017;89(3):291-301. doi:10.1212/WNL.0000000000004112

31. Bottiroli S, Allena M, Sances G, et al. Psychological, clinical, and therapeutic predictors of the outcome of detoxification in a large clinical population of medication-overuse headache: a six-month follow-up of the COMOESTAS project. Cephalalgia. 2019;39 (1):135-147. doi:10.1177/0333102418783317

32. Chen Z, Chen X, Liu M, Ma L, Yu S. Lower hippocampal subfields volume in relation to anxiety in medication-overuse headache. Mol Pain. 2018;14:2070402263. doi:10.1177/1744806918761257

33. Kruit MC, van Buchem MA, Launer LJ, Terwindt GM, Ferrari MD. Migraine is associated with an increased risk of deep white matter lesions, subclinical posterior circulation infarcts and brain iron accumulation: the population-based MRI CAMERA study. Cephalalgia. 2010;30(2):129-136. doi:10.1111/j.1468-2982.2009.01904.x

34. Bolandzadeh N, Davis JC, Tam R, Handy TC, Liu-Ambrose T. The association between cognitive function and white matter lesion location in older adults: a systematic review. Bmc Neurol. 2012;12:126. doi:10.1186/1471-2377-12-126

35. Lampe L, Kharabian-Masouleh S, Kynast J, et al. Lesion location matters: the relationships between white matter hyperintensities on cognition in the healthy elderly. J Cereb Blood Flow Metab. 2019;39 (1):36-43. doi:10.1177/0271678X17740501

36. Habes M, Sotiras A, Erus G, et al. White matter lesions: spatial heterogeneity, links to risk factors, cognition, genetics, and atrophy. Neurology. 2018;91(10):e964-e975. doi:10.1212/WNL.0000000000006116

37. De Groot JC, De Leeuw FE, Oudkerk M, et al. Periventricular cerebral white matter lesions predict rate of cognitive decline. Ann Neurol. 2002;52(3):335-341. doi:10.1002/ana.10294

38. Yang D, Huang L, Luo C, et al. Impaired structural network properties caused by white matter hyperintensity related to cognitive decline. Front Neurol. 2020;11:250. doi:10.3389/fneur.2020.00250

39. Bahrani AA, Powell DK, Yu G, Johnson ES, Jicha GA, Smith CD. White matter hyperintensity associations with cerebral blood flow in elderly subjects stratified by cerebrovascular risk. $J$ Stroke Cerebrovasc Dis. 2017;26(4):779-786. doi:10.1016/j.jstrokecerebrov asdis.2016.10.017

40. Fiford CM, Manning EN, Bartlett JW, et al. White matter hyperintensities are associated with disproportionate progressive hippocampal atrophy. Hippocampus. 2017;27(3):249-262. doi:10.1002/hipo.22690

41. Zi W, Duan D, Zheng J. Cognitive impairments associated with periventricular white matter hyperintensities are mediated by cortical atrophy. Acta Neurol Scand. 2014;130(3):178-187. doi:10.1111/ane.12262

42. Murman D. The impact of age on cognition. Semin Hear. 2015;36 (03):111-121. doi:10.1055/s-0035-1555115

43. Huang L, Juan DH, Wang X, Wang Y, Xiao Z. Duration and frequency of migraines affect cognitive function: evidence from neuropsychological tests and event-related potentials. J Headache Pain. 2017;18(1):54. doi:10.1186/s10194-017-0758-6 


\section{Publish your work in this journal}

The Journal of Pain Research is an international, peer reviewed, open access, online journal that welcomes laboratory and clinical findings in the fields of pain research and the prevention and management of pain. Original research, reviews, symposium reports, hypothesis formation and commentaries are all considered for publication. The manuscript management system is completely online and includes a very quick and fair peer-review system, which is all easy to use. Visit http:// www.dovepress.com/testimonials.php to read real quotes from published authors.

Submit your manuscript here: https://www.dovepress.com/journal-of-pain-research-journal 